\title{
PREVALENCE OF ENDOPARASITES IN CARNIVORES IN A ZOO AND A WOLVES PARK IN GERMANY
}

\author{
Schieber, M.-C., Štrkolcová, G. \\ Institute of Parasitology \\ University of Veterinary Medicine and Pharmacy in Košice \\ Komenského 73, 04181 Košice \\ Slovakia \\ gabriela.strkolcova@uvlf.sk
}

\section{ABSTRACT}

Endoparasites have the potential to cause significant health problems in humans and other animals. Consequently, particularly the endoparasites of a zoonotic nature are of great interest to health authorities and scientists. This study investigated the prevalence of different intestinal parasite species of carnivores kept in captivity. Altogether 36 pooled samples of faeces obtained from individual animal enclosures from the Neunkircher Zoo and Wolfspark Werner Freund in Germany were examined. The samples were analysed by means of a flotation concentration method with the use of Faust and KozákMágrová solution. Out of a total of 36 samples, 19 were positive for endoparasites $(\mathbf{5 2 . 7 8 \% )}$ ). Furthermore, 13 out of 19 positive samples were protozoans $(68.42 \%)$.

Key words: carnivores; endoparasites; wolves park; zoo

\section{INTRODUCTION}

Zoological gardens and animal parks display wild animals for recreational and educational purposes but also play an important role in the conservation of species, particularly the rare and endangered ones [18]. In their natural habitat, wild animals have large areas available to them. Their exposure to parasitic infections is, therefore, fairly low and they have consequently a low genetic resistance against parasitic infections. When groups of these wild animals are kept in confined space and many captive animal species are housed in close proximity to each other, the problem of parasitic infections can become aggravated and pose a serious threat to the animals; occasionally causing sudden local fatalities [16].

The occurrence of parasites in animals housed in zoos varies according to the type of husbandry, parasite prophylaxis and type of parasitic treatments. Usually, captive animals in zoos do not show alarming signs of parasitism if deworming is carried out regularly [18]. In addition, captivity animals are often under considerable stress, which 
further diminishes their resistance to parasitic infections. One should also consider the impact of environmental changes on the zoonotic disease risk, which is mostly the subject of speculation and lacks a coherent framework for understanding environmental drivers of pathogen transmission from animal hosts to humans [3].

Endoparasites have the potential to cause a significant health problem in humans and other animals. Particularly endoparasites with a zoonotic nature are of great interest to health authorities and scientists $[3,6]$.

In the study by $\mathrm{Kh}$ a $\mathrm{t} \mathrm{u}$ et al. [10], $60 \%$ of the animals (72.7\% of carnivores) at Rangpur Recreational Garden and Zoo in Bangladesh were found positive for gastrointestinal parasites. Other authors reported similar [18], higher $[5,17]$ or lower [14] prevalence, but the prevalence always ranged between 40.4 and $76.6 \%$. In all animals, except primates, the prevalence of helminth infections was higher than the prevalence of protozoan infections, similar to other studies [18].

In this study, faecal samples of carnivores from a zoo and a wolves park in Germany were investigated for intestinal parasites in order to obtain information on the prevalence of endoparasites in captive-held carnivores in these facilities. Such an assessment should involve the consequences the parasite has on the host itself, its transmission abilities and its role in public health [13]. As environmental factors play an important role in the occurrence of parasites [20], the cleaning and feeding regime of the animals should also be mentioned.

In the zoo, various species of carnivores were kept: yellow-throated martens (Martes flavigula), slender-tailed meerkats (Suricata suricatta), raccoons (Procyon lotor), snow leopards (Uncia uncia), asiatic wild dogs (Cuon alpinus) and bush dogs (Speothos venaticus).

The Wolfspark Werner Freund kept 4 Canadian wolves (Canis lupus lycaon), 4 Mongolian wolves (Canis lupus chanco), 3 Swedish wolves (Canis lupus lupus) and 10 arctic wolves (Canis lupus arctos), which were divided into one 4-wolf and two 3-wolf packs. All of the groups had their separate enclosures, spread across 7 hectare of the forest. A pronounced focus of this park is to keep the animals under as natural conditions as possible.

An important difference between the animals in the zoo and the wolves park is, that the zoo animals have contact with a higher number of different people (zoo keepers), while the wolves are only in direct contact with two people.
Another difference is that the zoo animals receive anti-parasitic treatment every spring and autumn while the wolves are treated with anti-parasitic as puppies and as adults, they will only receive such treatment when specific symptoms indicate a higher infestation with parasites, causing health problems to the animals.

The aim of the study was to get an impression of the current endoparasitic situation in relevant and often-overlooked animals, as those can potentially reflect a zoonotic risk. As a result, a more precise anti-parasitic treatment can be instituted, helping to protect the environment, the animals and counteract resistance development against antiparasitic products, hence improving the management of the endoparasites.

\section{MATERIALS AND METHODS}

In this study, 36 pooled faecal samples of carnivores from the Neunkircher Zoo and the Wolfspark Werner Freund in Merzig in Germany were investigated for intestinal parasites.

\section{The feeding and cleaning regimens}

The yellow-throated marten was fed a diet composed of small mammals, birds, insects, nuts and fruits, once a day. The enclosure was cleaned every $2-3$ days. The slendertailed meerkats received three times a day mainly an insectivorous diet with an addition of small mammals and some plant material. Their enclosure was cleaned once a day. The raccoons were fed once a day, mainly an insectivorous and plant based diet with fruits and nuts. Occasionally, also fish and eggs might be included in their diet plan. As with the martens, the enclosure was cleaned every $2-3$ days. The snow leopards were fed once a day as other solitary mammals and birds. They were not fed one day a week. The enclosure was cleaned once a week. The pack of Asiatic wild dogs were fed mainly mammals. Additionally, they also receive some insects and fruits. As with snow leopards, they were fed once a day, while they did not receive any food for one day a week. Their enclosure was cleaned every $1-2$ weeks. The bush dogs were fed mammals, particular rodents and birds. As with the previous 2 animals, they did not receive any food one day a week and their enclosure was cleaned every week once.

The wolves were kept as close to their natural condi- 
tions so they were not fed every day. The time span between their meals depended on the size of the previous meal. They received animals from hunters and forest rangers (mainly deer), farmers (cattle, sheep, goats) and some local privately owned animals such as horses. Pigs were not fed.

\section{Collection and processing of samples}

The samples were collected in April 2017 and November 2017. Multiple samples were collected from each individual enclosure in a plastic bag and stored individually at $4{ }^{\circ} \mathrm{C}$. The pooled samples were evaluated within 24 hours. For analysis of all samples, we used the flotation concentration test with zinc sulphate solution (Faust solution-specific gravity $1.18{\mathrm{~g} . \mathrm{cm}^{-3}}^{-3}$ for the presence of protozoan cysts (Giardia duodenalis) and Kozák-Mágrová solution for the presence of protozoan oocysts and helminth eggs [12].

For the flotation concentration method with zinc sulphate solution we mixed 5-10 grams of a composite faecal sample with $20-30 \mathrm{ml}$ of water to make a semi-solid suspension. The suspension was filtered through a plastic sieve into a beaker and the filtrate was transferred to two tubes which were centrifuged for $2 \mathrm{~min}$ at $2500 \mathrm{rpm}$. Subsequently, the supernatant was poured off from both tubes. To the remaining pellet on the bottom of one tube we added Faust solution (zinc sulphate solution) up to $1 / 3$ of the tube volume and to the second tube we added Kozák-Mágrová solution. The content of both tubes were mixed and centrifuged at the same setting as above. Using a parasitological loop, we picked up 3 drops from the solution surface, transferred them onto a clear glass slide and examined under a light microscope Olympus Model BX41 (Japan). For detection of cysts of Giardia duodenalis the drops were covered with a cover slip and also observed under a light microscope.

\section{RESULTS}

Out of a total of 36 samples, 19 were positive for endoparasites $(52.78 \%)$. The results are summarized in Table 1. Thirteen out of 19 positive samples were of protozoan ori$\operatorname{gin}(68.42 \%)$.

Out of 6 samples from the yellow-throated martens, 1 was positive for Ancylostoma spp. (Fig. 1). All 6 samples from the slender-tailed meerkats, were positive for Cystoisospora spp. From the 6 samples obtained from raccoons, 3 were positive for Cystoisospora spp. (Fig. 2). The 5 samples from the snow leopard, included 3 positive samples for Toxocara cati (Fig. 3) and 1 for Ancylostoma spp. Of the 3 samples from the asiatic wild dog, 2 were positive for Sarcocystis spp. Two samples originated from the bush dog (Speothos venaticus) and both were positive for Sarcocystis spp. From a total of 8 samples obtained from wolves only 1 sample was positive for Trichuris vulpis.

Table 1. Number of positive samples for each animal and species of endoparasites found

\begin{tabular}{lccc}
\hline \multicolumn{1}{c}{ Animal Species } & Number of samples & $\begin{array}{c}\text { Number } \\
\text { of positive samples }\end{array}$ & $\begin{array}{c}\text { Species } \\
\text { of endoparasites }\end{array}$ \\
\hline $\begin{array}{l}\text { Yellow-throated marten } \\
\text { (Martes flavigula) }\end{array}$ & 6 & 1 & Ancylostoma spp. \\
$\begin{array}{l}\text { Slender-tailed meerkat } \\
\text { (Suricata suricatta) }\end{array}$ & 6 & 6 & Cystoisospora spp. \\
$\begin{array}{l}\text { Raccoon } \\
\text { (Procyon lotor) }\end{array}$ & 6 & 3 & Cystoisospora spp. \\
$\begin{array}{l}\text { Snow leopard } \\
\text { (Uncia uncia) }\end{array}$ & 5 & 3 & Toxocara cati \\
$\begin{array}{l}\text { Asiatic wild dog } \\
\text { (Cuon alpinus) }\end{array}$ & 3 & 1 & Ancylostoma spp. \\
$\begin{array}{l}\text { Bush dog } \\
\text { (Speothos venaticus) }\end{array}$ & 2 & 2 & Sarcocystis spp. \\
$\begin{array}{l}\text { Wolves } \\
\text { (Canis lupus) }\end{array}$ & 8 & 2 & Sarcocystis spp. \\
\hline TOTAL & 36 & 1 & Trichuris vulpis \\
\hline
\end{tabular}




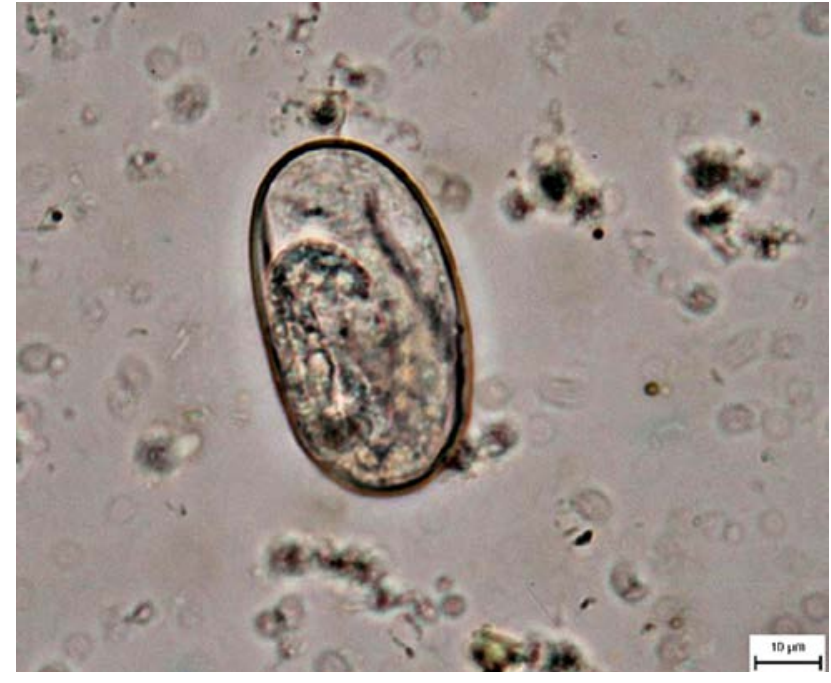

Fig. 1. Ancylostoma sp. egg with larva in a yellow-throated marten. Magn. $\times 100$

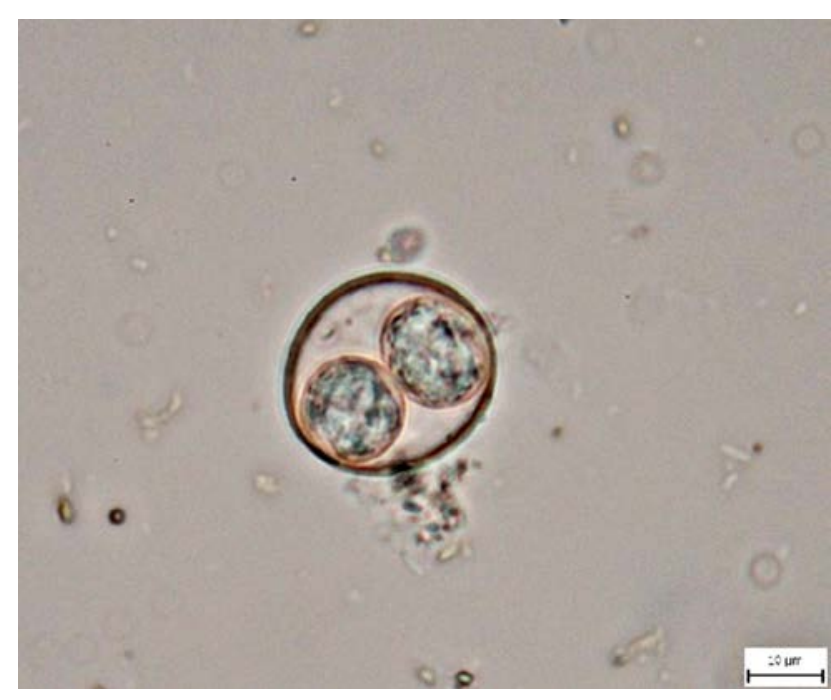

Fig. 2. Cystoisospora sp. oocyst in a raccoon. Magn. $\times 100$

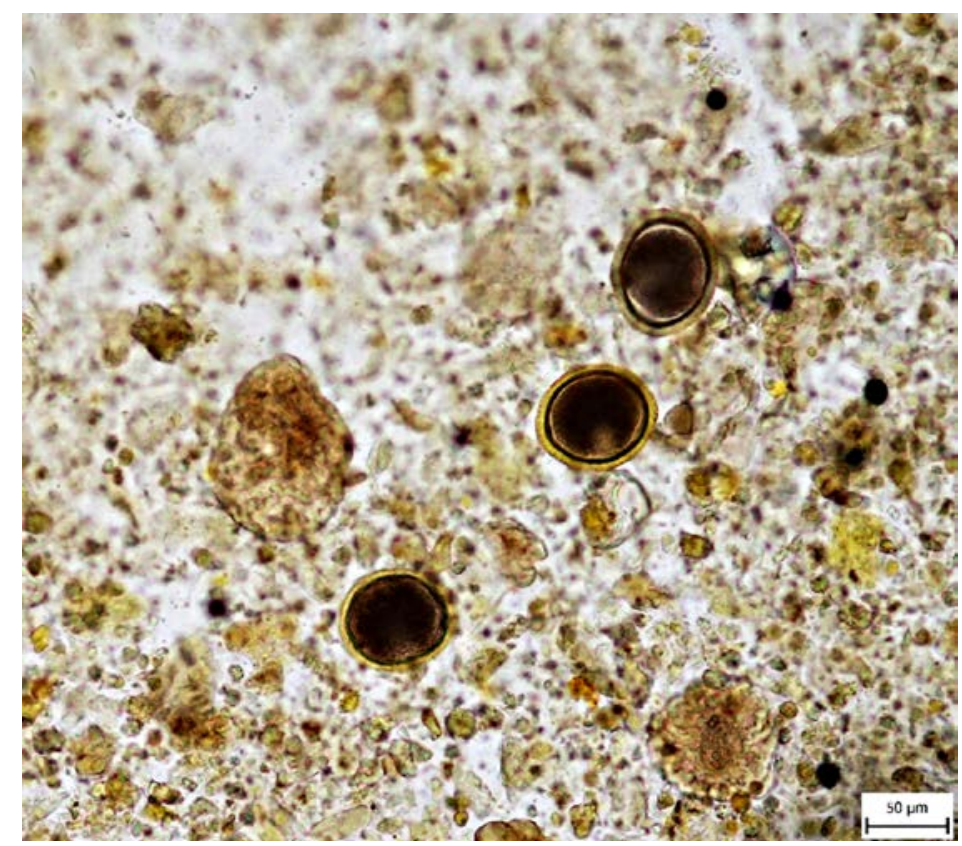

Fig. 3. Toxocara cati eggs in a snow leopard. Magn. $\times 20$

\section{DISCUSSION}

A total of $52.78 \%$ of the samples were positive for one or more endoparasitic species, from which $68.42 \%$ belonged to protozoa. In the study by $\mathrm{Kh}$ a t u n et al. [10] $72.7 \%$ of the carnivores were found positive for gastrointestinal parasites. Higher (97.3\% and $89.3 \%$ ) and lower (50\%) infection rates were found by other authors $[14,15])$.

It is interesting to notice that all samples from the meer- kats were positive for Isospora spp. This can be explained by the fact that in general higher occurrences of this parasite appear in younger animals $[8,11]$, which correlates with the constitution of the clan, as more than half were only 14 months old at the time of the collection of the samples.

With regard to the zoonotic risk, particularly Ancylostoma spp., Toxocara spp. and Sarcocystis spp. should be noticed. Ancylostoma spp. is commonly known as a hookworm. Pathogenesis arises from mature adults, grasping 
plugs of intestinal mucosa with their toothed buccal capsule, to feed from blood (approximately $0.1 \mathrm{ml} \mathrm{blood} /$ day) [22]. Pathogenesis is displayed as acute or chronic haemorrhagic anaemia, particularly in young animals as older animals can often compensate for the anaemia to a certain degree. Yet, after a prolonged period of time, the animal will experience iron deficiency, leading to microcytic hypochromic anaemia [22].

Also some species such as Ancylostoma braziliens and Ancylostoma caninum pose a zoonotic risk, causing eosinophilic enteritis and cutaneous larva migrans in humans [22]. Humans can be infected via the third stage larvae (filariform), penetrating the skin [19].

Toxocara spp. can be found worldwide in the small intestine of its final host. Humans can become a dead end host, resulting in larva migrans visceralis. Commonly affected is the liver, resulting in hepatomegaly and eosinophilia [21]. If larvae escape the circulation, they can end up in other organs, notably the eye, commonly referred to as larva migrans ocularis [21]. The body's immune system will respond with proliferative inflammation, resulting in granuloma formation around the larvae, typically sitting on the retina, rarely on the optic disc. While a total loss of vision is uncommon, partial loss with endophthalmitis or granulomatous retinitis is more commonly reported [21].

Humans can serve as a final host in Sarcocystis hominis and S. suihominis, when consuming undercooked, infected meat. Additionally, humans can also function as intermediate host for S. nescbitti by ingesting faecally contaminated food, water or simply from the environment. Clinical signs might appear as headaches, fever and myalgia [7].

We detected a low occurrence of parasites in the wolves park compared to the zoo. As the number of samples was low, such a trend might be simply due to the chance of parasites shedding irregularly or only in small numbers at the time, and thus they might not have been detected [1]. Yet, it could also be explained by the less confined environment of the wolves, compared to the zoo [2]. For the zoo animals might be more likely to re-infect themselves after treatment, particularly if the enclosures are not properly cleaned after administration of the drugs. On the other hand, the wolves were in closer contact with wild animals (birds, rodents), which they regularly eat. However, they did not receive regular anti-parasitic treatments, which speaks against such a trend. The decreased use of prophy- lactic anti-parasitic drugs, which would in the long term result in the development of resistance to antiparasitics [4, 9], might explain the observed trend.

For future study, it would be interesting to investigate the parasitic burden of the zoo animals before and after the treatment in order to investigate the current resistance status of the parasites on the property.

\section{CONCLUSIONS}

Despite the low number of samples, the data can be used to compare the changes in endoparasite burden with regard to species and annual occurrence. This can be expected due to climate change and agricultural influences. Hence, regular epizootiological investigations of captive wild animals will help to increase our insight of not only the prevalence of such parasites but also its transmissibility between wild as well as domesticated animals and humans.

\section{ACKNOWLEDGEMENTS}

This study was supported by the project VEGA No. 1/ 0536/18 and Neunkircher Zoo and Wolfspark Werner Freund in Merzig.

\section{REFERENCES}

1. Al-Sabi, M. N. S., Kapel, Ch. M. O., Johansson, A., Espersen, M. C., Koch, J., Willesen, J. L., 2013: A coprological investigation of gastrointestinal and cardiopulmonary parasites in hunting dogs in Denmark. Vet. Parasitol., 196 (3-4), 366-372.

2. Arneberg, P., Skorping, A., Grenfell, B., Read, A. F., 1998: Host densities as determinants of abundance in parasite communities. Proceedings of the Royal Society of London B: Biological Sciences, 265 (1043), 1283'1289. DOI: 10.1098/rspb. 1998-0431.

3. Batchelor, D. J., Tzannes, S., Graham, P. A., Wastling, J. M., et al., 2008: Detection of endoparasites with zoonotic potential in dogs with gastrointestinal disease in the UK. Transboundary and Emerging Diseases, 55 (2), 99-104. DOI: 10.1111/j.1865-1682.2007.01005.x. 
4. Chapman, H. D., 1997: Biochemical, genetic and applied aspects of drug resistance in Eimeria parasites of the fowl. Avian Pathology, 26 (2), 221-244. DOI: 10.1080/ 03079459708419208

5. Corden, P., Prados, G. H., Romero, A., Sanchez, M. S., Pontes, M., Osuna, A., Rosales, M. J., 2008: Intestinal parasitism in the animals of the zoological garden "Peña Escrita" (Almuñecar, Spain). Vet. Parasitol., 156 (3-4), 302-309. DOI 10.1016/j.vetpar.2008.05.023

6. Estrada-Peña, A., Ostfeld, R. S., Peterson, A. T., et al., 2014: Effects of environmental change on zoonotic disease risk: an ecological primer. Trends in Parasitology, 30 (4), 205-214. DOI: 10.1016/j.pt.2014.02.003.

7. Fayer, R., Esposito, D. H., Dubey, J. P., 2015: Human infections with Sarcocystis species. Clinical Microbiology Reviews, 28 (2), 295-311. DOI: 10.1128/CMR.00113-14.

8. Harleman, J. H., Meyer, R. C., 1983: Isospora suis infection in piglets. A review. Veterinary Quarterly, 5 (4), 178-85. DOI 10.1080/01652176.1983.9693894.

9. Hennessy, D. R., 1994: The disposition of antiparasitic drugs in relation to the development of resistance by parasites of livestock. Acta Tropica, 56 (2-3), 125-141. DOI: 10.1016/ 0001-706X(94)90059-0.

10. Khatun, M. M., Begum, N., Al Mamun M. A., Mondal, M. M. H., Shakif-Ul-Azam, M., 2014: Coprological study of gastrointestinal parasites of captive animals at Rangpur Recreational Garden and Zoo in Bangladesh. Journal of the Threatened Taxa, 6, (8), 6142-6147. DOI: 10.11609/JoTT. o3093.6142-7.

11. Khodakaram-Tafti, A., Mansourian, M., 2008: Pathologic lesions of naturally occurring coccidiosis in sheep and goats. Comparative Clinical Pathology, 17 (2), 87-91. DOI: 10.1007/ s00580-008-0719-1.

12. Letková, V., Čisláková, L., et al., 2010: Laboratory Diagnostic Methods in Veterinary Parasitology (In Slovak). University of Veterinary Medicine and Pharmacy in Košice, Slovakia, $110 \mathrm{pp}$
13. Liatis, T. K., Monastiridis, A. A., Panagiotis, B., et al., 2017: Endoparasites of wild mammals sheltered in wildlife hospitals and rehabilitation centres in Greece. Frontiers in Veterinary Science, 4, 220. DOI: 10.3389/fvets.2017.00220.

14. Lim, Y. A. L., Ngui, R., Shukri, J., Rohela, M., Mat, N. H. R., 2008: Intestinal parasites in various animals at a zoo in Malaysia. Vet. Parasitol., 157 (1-2), 154-159; DOI: 10.1016/j. vetpar.2008.07.015.

15. Muller-Graf, C. D., 1995: A coprological survey of intestinal parasites of Wild Lions (Panthera leo) in the Serengeti and the Ngorongoro crater. J. Parasitol., 81(5), 812-814.

16. Muoria, P. K., Muruthi, P., Rubenstein, D., Oguge, N. O., Munene, E., 2005: Cross-sectional survey of gastro-intestinal parasites of Grevy's zebras in southern Samburu, Kenya. African Journal of Ecology, 43, 392-395. DOI: 10.1111/ j.1365-2028.2005.00588.x.

17. Opara, M. N., Osuji, C. T., Opara, J. A., 2010: Gastrointestinal parasitism in captive animals at the zoological garden, Nekede Owerri, southeast Nigeria. Report and Opinion, 2 (5), $21-28$

18. Parsani, H. R., Momin, R. R., Maradin, M. G., Veer, S., 2001: A survey of gastrointestinal parasites of captive animals at Rajkot municipal corporation zoo, Rajkot, Gujarat. Zoos Print Journal, 16 (10), 604-606. DOI: 10.11609/JoTT.ZPJ.16. 10.604-6.

19. Prociv, P., Croese, J., 1996: Human enteric infection with Ancylostoma caninum: hookworms reappraised in the light of a "new" zoonosis. Acta tropica, 62, 23-44. DOI: 10.1016/ S0001-706X(96)00016-2.

20. Sures, B., 2004: Environmental parasitology: relevancy of parasites in monitoring environmental pollution. Trends in Parasitology, 20 (4), 170-177. DOI: 10.1016/j.pt.2004.01.014.

21. Taylor, M. A., Coop, R. L., Wall, R. L., 2007: Veterinary Parasitology, 3rd edn., Wiley-Blackwell, 874 pp.

22. Urquhart, G. M., Armour, J., Duncan, J. L., Jennings, F. W., Dunnet, A. M., 1996: Veterinary Parasitology, 2nd edn., Oxford U. K., Blackwell Science, 307 pp.

Received January 16, 2019

Accepted March 12, 2019 